INPE ePrint: sid.inpe.br/yolanda/2004/09.27.11.43 v1 2004-09-28

\title{
On Orbit Spatial Resolution Estimation of CBERS-1 CCD Camera
}

\author{
Kamel Bensebaa \\ Doctorate Student \\ National Institute for Space \\ Research - INPE \\ São José dos Campos, Brazil \\ camel@dpi.inpe.br
}

\author{
Gerald J. F. Banon \\ Senior Researcher \\ National Institute for Space \\ Research - INPE \\ São José dos Campos, Brazil \\ banon@dpi.inpe.br
}

\author{
Leila M. G. Fonseca \\ Senior Researcher \\ National Institute for Space \\ Research - INPE \\ São José dos Campos, Brazil \\ leila@dpi.inpe.br
}

\begin{abstract}
The first China-Brazil Earth Resources Satellite (CBERS-1) launched in 1999 has been developed by China and Brazil. It carries on-board a multisensor payload with different spatial resolutions called: Wide Field Imager (WFI), High Resolution CCD Camera (CCD) and Infrared Multispectral Scanner (IR-MSS). The performance of these sensors can be evaluated through the Point Spread Function (PSF) that enables an objective assessment of the spatial resolution. This work describes an approach to estimate on-orbit CBERS-1 CCD spatial resolution using an image of a black square target on the Gobi desert (China). The results show that the spatial resolution in across-track direction is not complied to the design specification for all bands whereas the spatial resolution in along-track direction is conform to the specification for all bands, except the band 4.
\end{abstract}

\section{Introduction}

The amount of satellite imagery has widely increased with the advent of new sophisticated imaging systems onboard new generation satellites. These systems have provided high quality data and have allowed a more accurate understanding of phenomena on the ground. Among this new generation of satellites, the CBERS-1 (China-Brazil Earth Resources Satellite) jointly developed by Brazil and China carries onboard three sensors that combine features that are specially designed to cover the broad range of space and time scales involved in the monitoring and preservation of the ecosystem. CBERS-1 was substituted by CBERS-2 launched on October 21, 2003, which is technically identical to CBERS-1. The imaging system performance usually changes on-orbit as a result of exposure to the space environment that might degrade its spatial resolution (blurring effect). Furthermore, Band 4 (0,77 $0,89 \mu \mathrm{m})$ of the CBERS-1 CCD camera presented additional spatial resolution degradation due to a problem in the camera assembly. In order to evaluate this spatial resolution degradation, the PSF (Point Spread Function) or MTF (Modulation Transfer Function) have been estimated [8].

Modelled as a Gaussian function, the PSF or MTF can be parameterized in terms of the EIFOV (Effective Instantaneous Field of View) which is a common measure used to evaluate the spatial resolution [2].

Storey [7] has provided a methodology to measure the Landsat-TM on-orbit spatial response using ground target such as bridges. Choi [3] has used airport runway and a tarp placed on the ground as targets to estimate the on-orbit Modulation Transfer Function (MTF) of IKONOS sensor.

This paper describes an approach to estimate the spatial resolution of the CBERS-1 CCD camera. The method estimates the along-track and across-track PSF of the CCD camera using an image of a simulated black squared target on the Gobi desert (China). The CCD spatial response is modeled as a 2D Gaussian function which is characterized by two parameters: one in alongtrack direction and another one in across-track direction. Therefore, the EIFOV values are derived from these parameters.

\section{CBERS-1 overview}

The first China-Brasil Earth Resources Satellite (CBERS-1) was launched on 14 October 1999 by the Chinese launcher Long-March 4B, from the Tayuan Launch Center, in the Popular Republic of China. The CBERS-1 payload consists of three instruments called: CCD (Charge Coupled Device) camera, IR-MSS (Infrared MSS) and WFI (Wide Field Imager). The 
high-resolution CCD Camera has 4 spectral bands from visible light to near infrared (B1: $0.45 \sim 0.52 \mu \mathrm{m}$; B2:

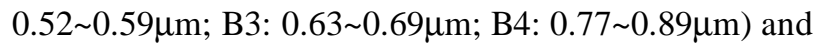
one panchromatic band (B5: $0.51 \sim 0.73 \mu \mathrm{m}$ ). It acquires the earth ground scenes by pushbroom scanning, on 778 $\mathrm{km}$ sun-synchronous orbit and provides images of 113 $\mathrm{km}$ wide strips with sampling rate of 20 meters at nadir. Since this camera has a sideways pointing capability of \pm 32 degrees, it is capable of taking stereoscopic images of a certain region.

The signal acquisition operates in two channels called CCD1 and CCD2. The first one generates images corresponding to Bands B2, B3 and B4 while the second one generates images corresponding to Bands B1, B3 and B5.

\section{PSF estimation methodology}

Basically, there exist three ways to estimate the system PSF. The first one uses images of targets with welldefined shape and size such as airport runway, bridges, etc or artificial target. The second method utilizes images acquired by higher resolution sensor, which are compared with the image under study. Finally, the third one uses the system design specifications and analytical model [4][5]. The method proposed in this study uses the first approach to estimate the system spatial resolution.

\subsection{Target images}

The target is an image of a dark squared target simulated on the Gobi desert. The Dunhuang test site of Gobi desert is situated at about $35 \mathrm{~km}$ west of Dunhuang city in Gansu Province, China. Its dimensions are about $30 \mathrm{~km}$ from south to north and $40 \mathrm{~km}$ from east to west. The size of the black target is 60 meters $\times 60$ meters, which corresponds to $3 \times 3$ pixels on the ground. Besides, the black target was aligned to the along track direction of the satellite (Fig. 1).

\subsection{Data preparation}

The target image was acquired by CBERS-1 on September 4, 2000. Fig. 2 shows the black target in the center of the image (Band4). In order to facilitate the visualization the image was zoomed up. For this experiment, only Bands B2, B3, and B4 were processed.

The raw images acquired by CBERS-1 system have a striping effect as shown on Fig. 2. This is due to the nonuniform detector gains, since each detector is responsible for one column in the image. The destriping procedure to remove the stripping effect consists of a linear pointwise radiometric correction. Figure 3 displays an image after the calibration process [1].

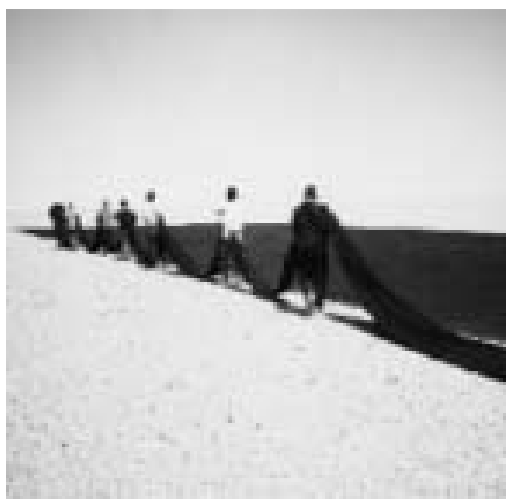

Figure 1. Setting up of black tarp in Gobi desert.

\subsection{Digital target model}

Let $\mathbf{Z}$ be the set of integer numbers and let $\mathbf{Z}^{\mathbf{2}}$ be the Cartesian product of $\mathbf{Z}$ by itself.

Let $x \in \mathbf{Z}$, we denote by $\underline{x}$ the pair $(x, x)$ of $\mathbf{Z}^{2}$. For example 10 stands for the pair $(10,10)$.

Let $F$ be a finite square of $\mathbf{Z}^{2}$ with an odd number of lines and columns representing the digital scene domain

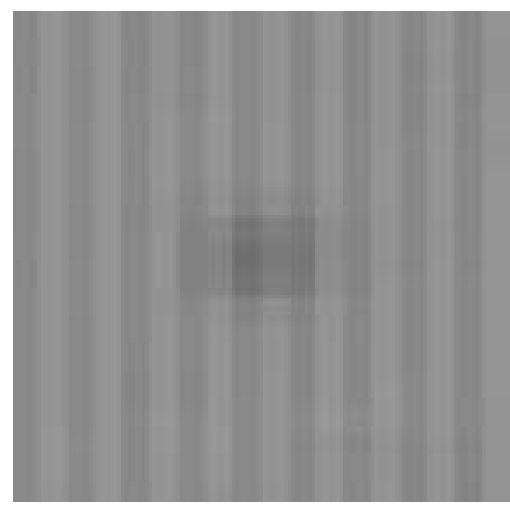

Figure 2. Original target band 3 image.

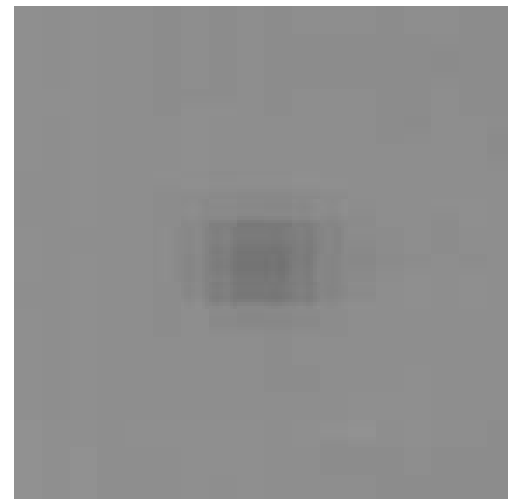

Figure 3: Target band 3 image after destriping. in which the distance between two consecutive horizontal or vertical points is one meter for convenience.

Let $u$ be the center point of $F$. Based on radiometric and geometric features of the target on the ground, the digital target model is the function $f$ on $F$ given by, for every $x \in F$, 


$$
f_{t}(x)= \begin{cases}t & \text { if } x \in[u-\underline{30}, u+\underline{30}] \\ s & \text { otherwise }\end{cases}
$$

where $s$ and $t$ are, respectively, the background (desert) and target radiometry values, and $[a, b]$ is the rectangle of $\mathbf{Z}^{2}$ having $a$ as lower left corner and $b$ as upper right corner. We observe that the digital target model is centered at $u$ and the target size is $60 \mathrm{~m} \times 60 \mathrm{~m}$ (Fig. 4).

\subsection{CCD point spread function}

The CBERS-1 CCD point spread function is modeled as a 2D Gaussian function $h_{\sigma_{1}, \sigma_{2}}$ on $F$, with center at $u$, that is, for every $\left(x_{1}, x_{2}\right) \in F$,

$$
h_{\sigma_{1}, \sigma_{2}}\left(x_{1}, x_{2}\right)=\frac{1}{2 \pi \sigma_{1} \sigma_{2}} e^{-\left(\frac{\left(x_{1}-u\right)^{2}}{2 \sigma_{1}^{2}}+\frac{\left(x_{2}-u\right)^{2}}{2 \sigma_{2}^{2}}\right)}
$$

\subsection{Target image simulation}

Let $G$ be a finite square of $\mathbf{Z}^{2}$ with an odd number of rows and columns, representing the target image domain.

Let $v$ be the center point of $G$ and let $T_{k}$ be a geometric transformation from $G$ to $F$ given by, for every $y \in G$,

$$
T_{k}(y)=20 .(y-v)+u+k
$$

where $k \in \mathbf{Z}^{2}$.

The transformation $T_{k}$ is the system geometric model, where the value 20 represents the distance (in meters) between two consecutive horizontal or vertical pixel positions (sampling rate). The offset $\mathrm{k}$ defines how far the transformation of the target image domain center $v$ is from the digital scene domain center $u$ (Fig. 4). By assuming that the imaging system is linear, the simulated target image is

$$
\left(f_{t} * h_{\sigma_{1}, \sigma_{2}}\right) \circ T_{k}
$$

where $*$ is the (circular) convolution product ( $u$ is being chosen as the origin) on $F$, and $\circ$ is the mapping composition. By composition definition, we observe that the simulated target image is a function on $G$.

\subsection{Point Spread function estimation}

Let $g$ be the target image defined on $G$, such that $g(v)$ has the lowest (recall that the target is black) value among all the pixel values of $g$. The PSF estimation consists of finding $\sigma_{1}$ and $\sigma_{2}$ such that $g$ and $\left(f_{t} * h_{\sigma_{1}, \sigma_{2}}\right) \circ T_{k}$ best fits under the root mean square criteria. Let $\operatorname{RMS}\left(f_{t}, g\right)$ be the real number given by

$$
\operatorname{RMS}\left(f_{t}, g\right)=\left(\sum_{y \in G}\left(\left(f_{t} * h_{\mathbf{\sigma}_{1}, \mathbf{\sigma}_{2}}\right)\left(T_{k}(y)\right)-g(y)\right)^{2}\right)^{1 / 2} .
$$

The PSF estimation is a two-steps procedure. At the first step, $t=g(v)$ and one looks for $k, \sigma_{1}$ and $\sigma_{2}$, which minimizes $\operatorname{RMS}\left(f_{t}, g\right)$.

Because $g(v)$ is the lowest value among the pixel values of $g$, the domain of $k$ reduces to $[-10, \underline{10}]$ of $\mathbf{Z}^{2}$. At the second step, one uses the previous optimum $k=\left(k_{1}\right.$, $k_{2}$ ) and one looks for $t, \sigma_{1}$ and $\sigma_{2}$ which minimizes $\operatorname{RMS}\left(f_{t}, g\right)$. The block diagram of Fig. 5 illustrates the PSF estimation. Figure 6 and 7 show the along-track and across-track fitting between the original data and simulated data for Band 3. The desert radiometry $s$ was estimated by averaging surrounding desert pixel values. In the above procedure, we have used $F=[\underline{1}, \underline{241}], u=$ $121, G=[\underline{1}, \underline{11}], v=6$ and the target radiometry value $t$ was considered within the following interval of $\mathbf{Z}$ :

$$
[g(v)-2, g(v)+2]
$$

Finally, the optimal values of $\sigma_{1}$ and $\sigma_{2}$ were obtained by nonlinear programming [6]. Table 1 shows the optimal values in along-track and across-track direction: background radiometry, target radiometry, offset, standard deviation and the Effective Instantaneous Field of View (EIFOV) which is related to the standard deviation $\sigma$ by the relation: $\mathrm{EIFOV}=2.66 . \sigma[2]$.

Table 1: Estimated parameters.

\begin{tabular}{|c|c|c|c|c|c|c|c|c|}
\hline Bands & $s$ & $t$ & $k_{1}(\mathrm{~m})$ & $k_{2}(\mathrm{~m})$ & $\sigma_{1}(\mathrm{~m})$ & $\sigma_{2}(\mathrm{~m})$ & $\mathrm{EIFOV}_{1}(\mathrm{~m})$ & $\mathrm{EIFOV}_{2}(\mathrm{~m})$ \\
\hline B2 & 91.20 & 72.9 & 10 & -5 & 12.7 & 25.65 & 34 & 68 \\
\hline B3 & 142.9 & 108.7 & -1 & 8 & 11.92 & 25.6 & 32 & 68 \\
\hline B4 & 116 & 90 & -10 & -10 & 19,04 & 28,67 & 51 & 76 \\
\hline
\end{tabular}




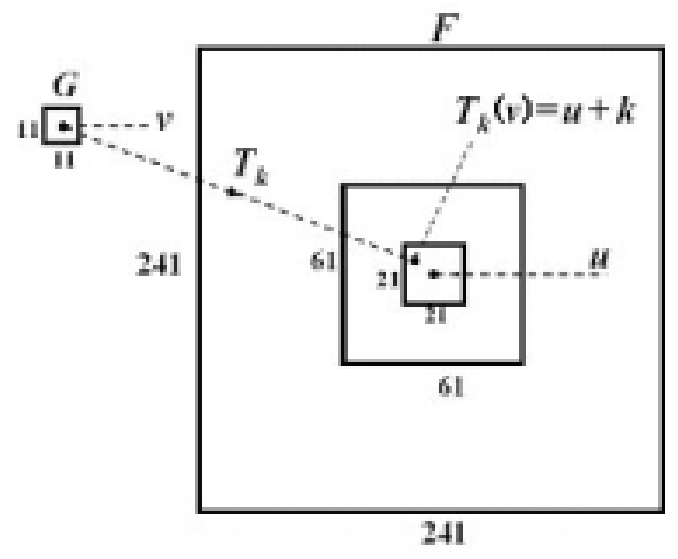

Figure 4: Digital target model.

\section{Conclusion}

An approach for the CBERS-1 CCD on-orbit PSF estimation has been introduced using images of a squared black target on the Gobi desert. The results show that the spatial resolution in across-track direction does not conform to the specification for all bands while the spatial resolution in along-track direction is complied to the specification for all bands, except band 4 .

The PSF estimated by the proposed method has been used to restore CBERS-1 images in order to improve their spatial resolution quality.

\section{References}

[1] G. J. F. Banon, Formal introduction to digital image processing. INPE-7682-PUD/097, National Institute for Space Research (INPE), São José dos Campos, Brazil 2000.<http://iris.sid.inpe.br:1905/rep/dpi.inpe.br/banon/1999/06.21.09.31>. Access in: 2003, Oct. 29.

[2] G. J. F. Banon and A. C. Santos, Digital filter design for sensor simulation: application to the Brazilian Remote Sensing Satellite. INPE-5523-RPQ/665, National Institute for Space Research (INPE), São José dos Campos, Brazil

1993.<http://iris.sid.inpe.br:1905/rep/dpi.inpe.br/banon/1 995/12.14.18.12>. Access in: 2003, Oct. 29.

[3] T. Choi and D. L. Helder, "Techniques for measuring the in-orbit modulation transfer function (MTF) for high spatial resolution imaging satellite," in Proc. High Spatial Resolution Commercial Imagery Workshop, Greenbelt, USA, March. 2001.

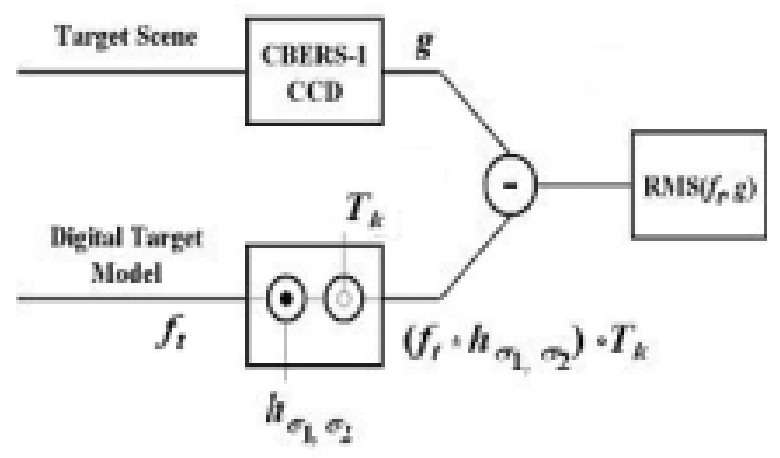

Figure 5: Block diagram of the PSF estimation.

[4] L. M. G. Fonseca, Determinação e avaliação das funções de transferência de modulação (MTF) dos sistemas MSS e TM (Landsat-5), INPE-4187-RPE/543, National Institute for Space Research (INPE), São José dos Campos, Brazil, 1987.

[5] L.M.G Fonseca and N. D. D. Mascarenhas, "Determinação da função de transferência do sensor TM do satélite Landsat-5," in Proc. Congresso Nacional de Matemática Aplicada e Computacional, Gramado, Brazil, Sept. 1987, pp. 221-224.

[6] D. M. .Himmelblau, Applied Nonlinear

Programming. New York: McGraw-Hill Book Company, 1972.

[7] J. C. Storey, "Landsat 7 on-orbit modulation transfer function estimation" in Proc. Sensors, Systems, and Next Generation Satellites V, Toulouse, France, 17-20 Sept. 2001, pp. 50-61.

[8] G. Wang and Y. Li, "Axiomatic approach for quantification of image resolution". IEEE Signal Processing Letters, 1999, v. 6, pp. 257-258. 